\title{
A cross-sectional study of factors affecting seasonality in bipolar disorder
}

\author{
P K Mittal, ${ }^{1}$ MD; S Mehta, ${ }^{2} \mathrm{MD} ; \mathbf{R}$ K Solanki, ${ }^{3} \mathrm{MD} ; \mathrm{M}$ K Swami, ${ }^{4} \mathrm{MD} ;$ P S Meena, ${ }^{2} \mathrm{MD}$ \\ ${ }^{1}$ Department of Psychiatry, All India Institute of Medical Sciences, Bhopal, Madhya Pradesh, India \\ ${ }^{2}$ Department of Psychiatry, Sawai Man Singh Medical College, Jaipur, Rajasthan, India \\ ${ }^{3}$ Department of Psychiatry, Dr Sampurnanand Medical College, Jodhpur, Rajasthan, India \\ ${ }^{4}$ Department of Psychiatry, BPS Government Medical College for Women, Sonepat, Haryana, India
}

Corresponding author: S Mehta (drshubhammehta@gmail.com)

Background. Researchers have evinced interest in the effect of seasonal variations on mood and behavioural patterns in affective disorders. Objective. To study seasonality in bipolar disorder (BD) patients and also the factors affecting this seasonality.

Method. Forty-nine patients with BD in euthymic phase were recruited and analysed using the Seasonal Pattern Assessment Questionnaire and Morningness-Eveningness Questionnaire.

Results. Most of the patients were morning types but chronotype had no influence on seasonality. Age of patient and number of episodes were the most important factors affecting seasonality in BD.

Conclusion. Seasonality and its influencing factors must be considered while managing bipolar disorder.

S Afr J Psych 2014;20(2):58-59. DOI:10.7196/SAJP.491

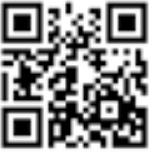

Seasonality in mood disorders was initially studied in the context of seasonal affective disorder (SAD), but later work has demonstrated that seasonality can be considered as a continuum and patients with mood disorders not diagnosed as SAD also have seasonal worsening of symptoms. ${ }^{[1-3]}$

Approximately $10 \%$ of all affective disorders show a seasonal pattern of recurrence. Half of these are recurrent depressive disorders; the other half are bipolar disorders (BDs).$^{[4]}$ It is important to identify seasonal changes in mood and behavioural pattern in patients with these disorders because seasonality affects prognosis and implies a more severe or complex disorder. ${ }^{[5]}$ Recognition of factors affecting seasonality also appears to be clinically relevant since these factors might predict recurrence of an affective episode. ${ }^{[6]}$

With the objective of assessing seasonality and its contributing factors in patients with $\mathrm{BD}$, we did a cross-sectional study involving 49 euthymic patients with BD diagnosed according to International Statistical Classification of Diseases and Related Health Problems (ICD)-10 criteria.

\section{Methods}

Patients were selected from those who came for follow-up visits to the outpatient Department of Psychiatry in Sawai Man Singh Hospital, Jaipur, India. Only those patients who had been euthymic for at least 24 months were recruited. Euthymia was defined according to a score of $<8$ on the Hamilton Depression Rating Scale-17 and a score of 0 on Young's Mania Rating Scale. Patients with any comorbid psychiatric illness and current or past history of substance abuse were excluded.

After informed consent was obtained for study participation, sociodemographic and clinical data were recorded on a author-designed Performa. A global seasonality score (GSS) with total scores ranging from 0 to 24 was derived from the Seasonal Pattern Assessment Questionnaire, after the patients graded the effect that changes of seasons had on them in six areas: sleep length, social activity, mood, weight, appetite and energy level. Higher GSSs indicated higher seasonality. ${ }^{[7]}$ Participants were also asked if they perceived any seasonal changes that they experienced as problematic or not.

Previous research has indicated that there may be a significant relationship between chronotype and mood seasonality. ${ }^{[8]}$ We therefore also analysed the circadian typology and chronotype of patients using the self-report Morningness-Eveningness Questionnaire (MEQ). ${ }^{[9,10]}$ The 'morningness type' applies to people who prefer diurnal activity and 'eveningness types' are those who prefer nocturnal activities. Responses to the questionnaire are known to be normally distributed in the general population. ${ }^{[1]}$ This observation allows us to consider the circadian types as a continuum. ${ }^{[12]}$ The sum of the coded responses yields a global score ranging from 16 to 86 , with lower scores indicating greater eveningness tendencies and higher scores indicating greater morningness tendencies.

Statistical analysis of data was done using the $\chi^{2}$ test and Pearson correlation with the help of SPSS version 17.

\section{Results}

Most patients in our study were Hindu, married males who were farmers and labourers, and who belonged to nuclear families with rural backgrounds. The most common level of education completed was middle school. Family income ranged between Rs6 000 and Rs15 000 (ZAR1 080 to ZAR2 700). The majority of participants (47\%) experienced moderate seasonality, followed by mild seasonality (41\%) and severe seasonality (12\%) when grouped by GSSs. Although no consistent pattern could be established between variables of seasonal change, such as weight, sleep, appetite, etc., more than half of the 
Table 1. Correlation of clinical variables

\begin{tabular}{lccccc}
\hline \multirow{2}{*}{ Variable } & \multicolumn{2}{c}{ GSSs } & & \multicolumn{2}{c}{ MEQ scores } \\
\cline { 2 - 3 } \cline { 5 - 6 } Age & $\boldsymbol{r}$-value & $\boldsymbol{p}$-value & & $\boldsymbol{r}$-value & $\boldsymbol{p}$-value \\
Episodes (total) & 0.390 & $0.006^{*}$ & & 0.197 & 0.174 \\
Episodes (mania) & 0.875 & $0.000^{*}$ & & -0.005 & 0.974 \\
Episodes (depression) & 0.932 & $0.000^{*}$ & & -0.033 & 0.822 \\
Duration of treatment & 0.274 & $0.004^{*}$ & & 0.029 & 0.841 \\
Duration of euthymia & 0.282 & 0.057 & & 0.068 & 0.640 \\
MEQ & -0.018 & 0.905 & & -0.037 & 0.800 \\
GSS $=$ global seasonality score; MEQ $=$ Morningness-Eveningness Questionnaire. \\
${ }^{*} p<0.01$.
\end{tabular}

patients $(53 \%)$ reported that the seasonal changes they experience were problematic for them. Of our total patients, $67 \%$ were of definite or moderate morning type, $33 \%$ were intermediate types and none were evening types, according to the MEQ. There was a significant correlation between seasonality and total number of affective episodes $(r=0.875, p=0.000)$, manic episodes $(r=0.932, p=0.0000)$, depressive episodes $(r=0.403, p=0.004)$ and age $(r=0.390, p=0.006)$ (Table 1). There was no significant correlation between GSSs and MEQ scores $(r=-0.018, p=0.905)$ nor between other sociodemographic variables of the subjects with different seasonality types and different chronotypes.

\section{Discussion}

Confirming the findings of several previous studies ${ }^{[1,3,5]}$ (with one exception), ${ }^{[7]}$ we found that patients with $\mathrm{BD}$ are sensitive to seasonal changes. Patients experiencing seasonal changes require special attention because they may need modifications in pharmacological and behavioural management strategies, as recommended by one of these recent studies. ${ }^{[5]}$

Interestingly, a 2007 study by Goikolea et al. ${ }^{[6]}$ found that patients with seasonal patterns of symptoms present predominantly with bipolar II disorder, have depressive onset, and exhibit depressive predominant polarity in their illness. The authors linked the presence of seasonal pattern with a predominantly depressive component.

It is possible that patients troubled with seasonal changes represent a subgroup of bipolar patients with higher disease burden, and that their biological characteristics make them more susceptible to seasonal influences and, in turn, BD.

Our study also confirms previous MEQ findings suggesting that $\mathrm{BD}$ patients are less likely to be evening types than the general population. ${ }^{10]}$ The morningness-eveningness dimension is regulated by a complex interaction between social, geographic and genetic factors. ${ }^{[1]}$ However, since research has suggested that these characteristics are strongly affected by age and culture, ${ }^{[13]}$ the findings of chronotype in our study should be viewed as a preliminary finding in our cultural setting until further information is available.

The positive correlation between seasonality and age indicates that, as age increases, seasonal sensitivity in patients with $\mathrm{BD}$ increases. This finding is similar to those in earlier studies that report a positive relationship between seasonality and age. ${ }^{[8]}$ This observation has prognostic and management implications for patients with $\mathrm{BD}$.

Our study has some methodological limitations, including: use of questionnaires that ask respondents to remember the past, which introduces possible recall bias; a small sample size; the cross-sectional study design; and the lack of control group for comparison. Also, it is important to consider the findings in the context of Indian climate and culture. Cross-cultural studies in different climatic conditions with a longitudinal study design in future might provide a better insight into this effect.

\section{Conclusions}

On the basis of our study findings, it can be concluded that BD patients exhibit significant mood and behavioural changes with seasonal variations. Increasing age and burden of illness (i.e. number of manic or depressive episodes) is associated with seasonality in this patient group. Although most patients in this study showed morningness tendencies, circadian typology and chronotype seem to bear no relation to seasonality in $\mathrm{BD}$. We suggest that these findings might prove useful in planning better management strategies for patients with $\mathrm{BD}$ and therefore improve clinical outcomes.

\section{References}

1. Brambilla C, Gavinelli C, Delmonte D, et al. Seasonality and sleep: A clinical study on euthymic mood disorder patients. Depress Res Treat 2012;Article ID 978962:6 pages. [http:// dx.doi.org//10.1155/2012/978962]

2. Kasper S, Kamo T. Seasonality in major depressed inpatients. J Affect Disord 1990;19(4):243 248. [http://dx.doi.org/10.1016/0165-0327(90)90101-D]

3. Mittal PK, Mehta S, Solanki RK, et al. A comparative study of seasonality and chronotype in unipolar mania v. bipolar affective disorder. German J Psychiatry 2013;16(4):124-129.

4. Faedda GL, Tondo L, Teicher MH, et al. Seasonal mood disorders: Patterns of seasonal recurrence in mania and depression. Arch Gen Psychiatry 1993;50(1):17-23. [http://dx.doi. org/10.1001/archpsyc.1993.01820130019004

5. Geoffroy PA, Bellivier F, Scott J, et al. Bipolar disorder with seasonal pattern: Clinical characteristics and gender influences. Chronobiol Int 2013;30(9):1101-1107. [http://dx.doi. org/10.3109/07420528.2013.800091]

6. Goikolea JM, Colom F, Martinez-aran A, et al. Clinical and prognostic implications of seasonal pattern in bipolar disorder: A 10 -year follow-up of 302 patients. Psychol Med 2007;37(11):1595-1599. [http://dx.doi.org/10.1017/S0033291707000864]

7. Avasthi A, Gupta N, Kulhara P, et al. Seasonality in affective disorders using SPAQ. Indian I Psychiatry 2003;45(3):166-173

8. Horne JA, Ostberg O. A self-assessment questionnaire to determine Morningness Eveningness in human circadian rhythms. Int J Chronobiol 1976;4(2):97-110.

9. Hakkarainen R, Johansson C, Kieseppä T, et al. Seasonal changes sleep length and circadian preference among twins with bipolar disorder. BMC Psychiatry 2003;3:6. [http://dx.doi. org/10.1186/1471-244X-3-6]

10. Chelminski I, Ferraro FR, Petros T, Plaud JJ. Horne and Ostberg questionnaire: A score distribution in a large sample of young adults. Pers Individ Dif 1997;23(4):647-652. [http:// dx.doi.org/10.1016/S0191-8869(97)00073-1]

11. Natale V, Cicogna P. Morningness-eveningness dimension: Is it really a continuum? Pers Individ Dif 2002:32(5):809-816. [http://dx.doi.org/10.1016/S0191-8869(01)00085-X]

12. Caci $\mathrm{H}$, Adan A, Bohle P, et al. Transcultural properties of the composite scale of morningness: The relevance of the 'morning affect' factor. Chronobiol Int 2005:22(3):523-540. [http:// dx.doi.org/10.1081/CBI-20006240]

13. Tonetti L, Fabbri M, Martoni M, Natale V. Circadian type and mood seasonality in adolescents. Psychiatry Clin Neurosci 2012:66(2):157-59. [http://dx.doi.org/10.1111/j.1440-1819.2011.02303.x] 